\title{
Ovidio Decroly: Educación de los sentidos y del cuerpo del niño anormal
}

\author{
Ovidio Decroly: abnormal Child's senses and body education
}

\author{
Alexánder Yarza \\ Lorena Maria Rodriguez
}

\section{Resumen}

Palabras clave: Pedagogía de anormales, cuerpo anormal, Ovidio Decroly, educación motriz y sensorial, Centros de interés, juegos educativos,

\section{Abstract}

En el marco del proceso de apropiación e institucionalización de la pedagogia de anormales en Colombia entre 1920-1940, se quiere mostrar una particularidad en relación con los métodos de enseñanza y educación de los sentidos de los cuerpos anormales. Se analiza parte de la obra de Ovidio Decroly, pedagogo de anormales y activo experimental (apropiado en Colombia durante el mismo periodo), mostrando cómo se configura la educación de los anormales a partir de la educación motriz, los centros de interés y los juegos educativos. La tesis es que con estos métodos de enseñanza se educa la corporalidad anormal marcando una discontinuidad con los métodos de educación fisica clásicos (basados en lo terapéutico, lo clínico, lo militar) y estableciendo nuevas formas de prácticas que instituyen unos poderes sobre esos cuerpos.

\footnotetext{
cuerpos mezclados, habilidades hápticas, actividad fisica adaptada.
}

\begin{abstract}
In the process of adaptation and institutionalization of abnormal children's pedagogy in Colombia from 1920 through 1940, a special feature is shown concerning to the senses and body education methods in abnormal children. Ovidio Decroly's work is analyzed partly in this article. He was an abnormal children's educator and an experimental researcher. His work was adopted in Colombia during the same period of time. It is also shown how it is configured the abnormal children's education from the mobility training, centers of interest and educational sports. This article presents a thesis which it is that the abnormal body is trained with this teaching method, and these methods go away from the classical physical education methods (which are based on therapeutic, clinical and military aspects) and establish new ferms of practices which set up some powers in those bodies.
\end{abstract}

Key words: $\quad$ Body and senses education, mobility training, educational sports, abnormal children

Fecha de recepción: 3 de noviembre de 2004 .

Fecha de aceptación: 16 de mayo de 2005.

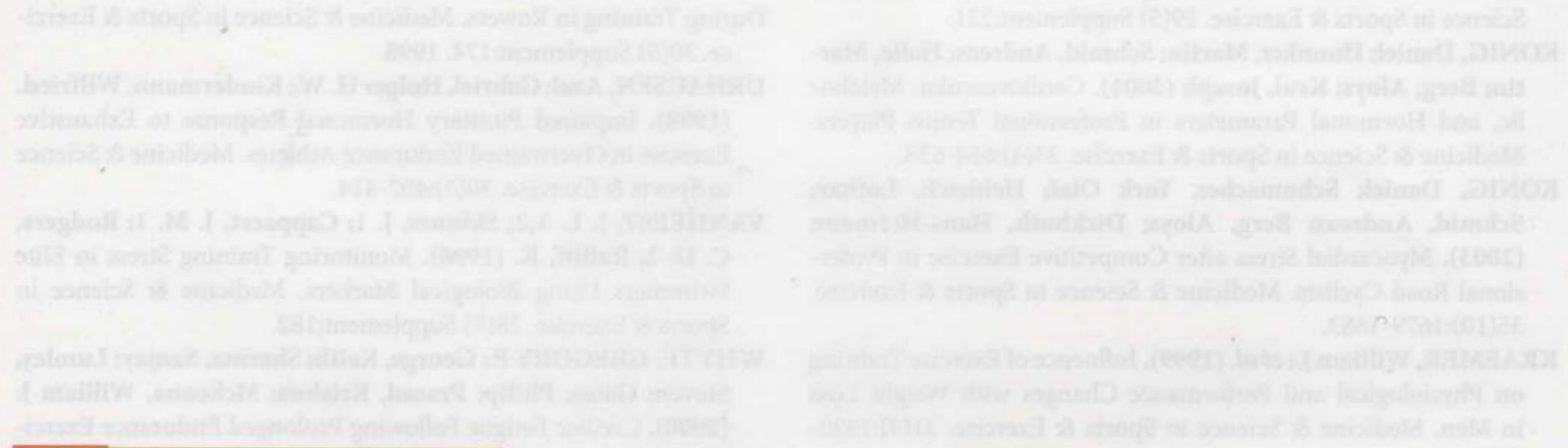

Este escrito es producto de la investigación en curso: El Rastro de la Diferencia. Historia de la Práctica Pedagógica de la Educación Especial en Colombia, 1845-1886, financiado por el Comité para el Desarrollo de la Investigación, Universidad de Antioquia -CODI-, 2002-2004. Es también adecuación de una ponencia presentada en el "Simposio Internacional Cuerpo, Motricidad y Desarrollo Humano. Una Mirada Transdisciplinar." Mayo 19 al 22. Medellín, Colombia. Conciencias, Instituto Universitario de Educación Física, Universidad de Antioquia.

*. Investigadores, Universidad Pedagógica Nacional 
Una vez graduado, se especializó en enfermedades nerviosas. Por sus consultas pasaron entonces varios anormales, que lo impresionaron profundamente, $y$ pronto se dio cuenta de que serian infructuosos los cuidados médicos que se prestasen a estos infelices si no eran continuados con la labor del maestro. Abrió camino para que el estado y la sociedad en general atendiesen a esta necesidad social; pues, aun considerados los anormales como escorias, ¿acaso las escorias no se aprovechan en la industria moderna de tal modo que nada desperdicia la economía industrial? Pues lo mismo debe suceder a la economía humana

Martín Restrepo Mejía: Pedagogo, maestro Pedagogia de párvulos, S. F., 1930, 123

Durante las décadas de 1920 a 1940 se institucionaliza en Colombia la educación o pedagogía de anormales. Los saberes que la legitiman y soportan son los siguientes: las "criminologías positivistas", la biomedicina, la psiquiatría, la psicología experimental, la pedagogía activa de corte experimental y los discursos eclesiásticos (moral católica). Las instituciones que reproducen y ponen en funcionamiento este discurso pedagógico son: Casa de Menores y Escuela de Trabajo San José (Fontidueño, Antioquia); Casa de Menores y Escuela de Trabajo Piedecuesta (Bucaramanga, Santander); Casa de Corrección y Escuela de Trabajo de Paiba (Cundinamarca); Escuela Especial Rafael Uribe Uribe, las Escuelas Activas de El Bosque y Los Libertadores, Escuela Sanín Cano, Instituto Médico Pedagógico, Escuela de Díscolos Tomás Cadavid Restrepo, y en cierta medida, la Escuela de Ciegos y Sordomudos, la Escuela Jesús María de Monfort para ciegas y sordomudas (Bello, Antioquia); el Instituto Nacional de Ciegos y Sordomudos (Bogotá, Cundinamarca). Los sujetos que se emplazan son: los maestros de anormales, los pedagogos especialistas, los médicos escolares y generales, los sacerdotes, los "jueces de menores" y los vigilantes (estos últimos en las Casas de Menores). Esta pedagógía pone en funcionamiento un conjunto de técnicas y prácticas que nombramos como tecnologías médico (psico) pedagógicas ${ }^{1}$, las cuales tienen la siguiente secuencia, serie o encadenamiento: prácticas o técnicas de observación (escolar, psicológica, antropométrica y biomédica), de clasificación, de registro (fichas médico-pedagógicas o formularios de observación), de etiologización, de diagnóstico, de selección, de ubicación o localización de niños anormales, y procedimientos de enseñanza especial.

En el marco de este proceso de apropiación e institucionalización, se quiere mostrar una particularidad en relación con los métodos de enseñanza y educación de los sentidos de los cuerpos anormales. Analizaremos parte de la obra de Ovidio Decroly, pedagogo de anormales y activo experimental (apropiado en Colombia durante el mismo período), mostrando cómo se configura la educación de los anormales a partir de la educación motriz, los centros de interés y los juegos educativos. La tesis es que con estos métodos de enseñanza se educa la corporalidad anormal marcando una discontinuidad con los métodos de educación física clásicos (basados en lo terapéutico o clínico y en lo militar) y estableciendo nuevas formas de prácticas que instituyen unos poderes sobre esos cuerpos. Al final, se deja la inquietud sobre las posibilidades de pensar una nueva educación física o sensorial de las corporalidades anormales contemporáneas, entendidas como cuerpos mezclados.

En primera instancia, el cuerpo anormal no se muestra, en todos los casos, en forma inmediata ante la mirada de quien observa. La visibilidad de este cuerpo extraño, no cotidiano, amorfo por demás deviene hacia la "luz" con la observación médico pedagógica o médico escolar, psicológica, biomédica y antropométrica. Una región de la mirada del saber pedagógico se encuentra subsumida por unas técnicas e instrumentos específicos que entrarán a funcionar a modo de filtro o tamiz para el pedagogo especialista, el maestro de anormales, el médico escolar, el maestro de escuelas especiales y el maestro de escuelas públicas.

La mirada del maestro de anormales sobre el cuerpo de sus estudiantes se comienza a formar (de formas) basada en instrumentos que sistematizan lo observado para modular (de modos) lo dicho. Las fichas médico-pedagógicas y los formularios médico-escolares se convierten en suplemento indispensable del ejercicio del ver. Estas fichas y formularios agrupan en distintas categorías las facultades, aptitudes, inclinaciones, vicios, instintos sexuales, principales gustos del niño, de su imaginación, de su conciencia moral si es hipócrita, arbitrario, autoritario-, de su atención, comparación, estigmas psíquicos.

Con esta observación y las fichas médico-pedagógicas parte de las tecnologías modernas de la pedagogía de anormales-se identifican los niños anormales, quienes son tomados como objeto de un conjunto de prácticas y tecnologías que determinarán, en parte, su espacio de encierro, el imaginario colectivo, sus aptitudes y dones naturales, la necesidad de ser útiles para la sociedad: su corporalidad anormal.

El cuerpo anormal emerge por la observación. Las condiciones y facultades mentales o intelectuales demarcan la anormalidad de los niños; estado que será generalizado a una subjetividad moderna en nacimiento y 
constitución: la infancia anormal (Álvarez Uría y Varela, 1991; Muel, 1991; Varela, 1995; Álvarez Uría, 1996; Herraiz, 1996; Cerezo, 2003). En tanto se forma, instruye y educa en lo físico, lo moral y lo intelectual; cualquier afección en una de estas dimensiones implica la modificación de las otras. Una anormalidad intelectual implicaría, por consiguiente, un cuerpo anormal: extraños brazos, gestos, estigmas en las caras, movimientos imprecisos, facciones estúpidas, orejas lánguidas, cráneos amorfos, vicios constitutivos; estas características se observaban en el juego, en el aula, en los patios, en los corredores.

Todo un retrato de miseria, degeneración y anormalidad se cierne sobre ese cuerpo. La pedagogía de anormales y la pedagogía activa entenderán que ejercitar lo manual, lo corporal, el cuerpo mismo, es un punto esencial para poder instruir y educar a los niños anormales. En este aspecto vital para estas pedagogias modernas se ancla parte de la obra pedagógica de Ovidio Decroly.

Ovidio Decroly nació en 1871 en Renaix (Bélgica) y murió en 1932 en una alameda del jardín del Instituto de Enseñanza Especial, cerca de sus pequeños niños irregulares $\mathrm{o}$ anormales.

[...] hizo sus estudios universitarios en la Facultad de Medicina de Gante en la que se doctoró [en 1896]; posteriormente obtuvo una beca para continuar sus estudios en Berlín y París. A su regreso fue nombrado en el servicio de neurología en Bruselas y jefe de servicios de niños retrasados de la policlínica. Su trabajo pedagógico se inicia propiamente en 1901 al fundar el instituto de enseñanza especial para retrasados y anormales. En 1907 crea la escuela del Ermitage para niños normales con el lema de "Escuela para la vida y por la vida" [une école pour la vie et par la vie]. En 1912 es nombrado profesor en cursos organizados para preparar profesores de enseñanza éspecial y así mismo se le designa como Director de la Sección de sicología. Posteriormente pasa a ser profesor del instituto superior de Pedagogía. En el mismo año funda la sociedad belga de Paidotecnia [...] Después de la guerra reanuda sus labores (1920) y es nombrado profesor de psicología del niño en la Universidad de Bruselas y profesor de higiene educativa y médico pedagógica en la Facultad de Medicina (Londoño Ramos, 2001, 136).

La experiencia que tuvo en la Policlínica de Bruselas fue decisiva en su construcción médico-pedagógica. "Confrontado con la miseria de las ciudades, Decroly descubrió el abandono humano, social y pedagógico en el que vegetaban sus pequeños pacientes. La escuela popular los condenaba casi siempre al fracaso y la mar- ginalización [...]" (Dubreucq, 1993, 261). Su método experimental aplicado, tanto en el Instituto de Enseñanza Especial como en la Escuela del Ermitage, pretendía resolver tres problemas esenciales: modificar la educación pasiva, memorística que imperaba en la instrucción, la necesidad de asegurar la enseñanza obligatoria a toda la infancia y el interés en generalizar métodos activos y experimentales en todas las escuelas, especiales y ordinarias. En esas escuelas-laboratorio se veía nacer una nueva pedagogía, de carácter práctico y activo, cuyo centro será el niño, tanto anormal como normal.

[...] Decroly está en todos los lugares donde lo requiere la infancia que sufre: funda, co-funda o anima el Hogar de Huérfanos, la Oficina de Orientación Profesional, la Granja-Escuela de Brabante, el Fondo de los Más Dotados de las Clases Populares y La Inspección Médica de la Infancia Delincuente. Las responsabilidades que asume y asigna a los educadores sobrepasan con creces los muros de las aulas: la "médico-pedagogía" (1904) implica la acción concertada del médico, el psicólogo, el docente y el asistente social, que trabajan en un sistema eficaz de orientación psico-médico-socio-escolar ( $\mathrm{Du}$ breucq, 1993, 264)

Su pedagogía es biológica, pragmatista, diferenciadora, activa y globalizadora. Toma la vida como base y fin, la utilidad como excusa y objetivo, la actividad del niño como centro, la globalidad y la integralidad como transversal a la enseñanza ${ }^{2}$. Tiene como trasfondo teórico el regeneracionismo y el reformismo social de la época. “[...] Decroly [...] afirmaba que en la lucha contra la degeneración y sus múltiples consecuencias la intervención del médico debe ser profiláctica y terapéutica a la vez, y el concepto terapéutico implica tratamiento médico y tratamiento pedagógico". (Varela, 1995, 174).

Decroly visita Colombia en 1925 , invitado por don Agustín Nieto Caballero a su Gimnasio Moderno, realizando conferencias y formando maestros de varias partes del país en su pedagogía. Durante las décadas de 1920 y 1930 se difunden sin cesar las ideas y métodos por todos los departamentos de la república. En 1935 se institucionalizan los centros de interés en el país. Durante más de dos décadas la educación pública y la escuela activa en Colombia estuvieron fuertemente influenciadas por la pedagogía de corte experimental de este pedagogo de anormales.

Para el caso que nos interesa, se rescatarán los aportes que este ilustre pedagogo belga formuló para la educación sensorial del niño anormal. Educación de los sentidos, de la sensibilidad que ponía como objetivo satisfacer las necesidades básicas o elementales en tanto 
posibilitan la vida material y mental al tiempo que favorecen el desarrollo del trabajo, suelo indudable de una pedagogía centrada en las necesidades, los medios, el interés, el aprendizaje, la actividad y el niño.

El cuerpo se convertirá en núcleo indiscutible de la pedagogía activa experimental decrolyana, el cual será entendido, principalmente, desde el positivismo evolucionista de Darwin y Spencer, es decir, como organismo vivo, adaptable, en evolución. Estará demarcado por los saberes modernos (psiquiatría, psicología experimental, sociobiología especulativa, medicina legal, etc.) apropiados a principios del siglo XX, y será fuertemente modificado por los influjos de la moral biológica y la moral católica: el hombre no puede existir sin Dios y sin alma. En otras palabras, el cuerpo será básico, pilar, central pero no exclusivo; la mente y el alma son elementos indispensables a considerar en su educación. Poder pedagógico sobre el cuerpo, la mente, el cerebro y el alma. Una escuela por y para la vida centrada en un principio de arduo moldeamiento del cuerpo anormal mediante múltiples métodos de enseñanza especial.

\section{Decroly y la educación del cuerpo} anormal

La educación física de los sentidos y del cuerpo anormal tiene tres formas de aparecer en Decroly: 1. Con la educación motriz y sensorial; 2 . Con los Centros de Interés; y 3. Con los juegos educativos. Los métodos Decrolyanos se sostienen en un mismo argumento: toman en cuenta las necesidades e intereses naturales del niño anormal y del niño normal. Naturalización que es producto del ejercicio del psicopoder (Varela, 1995: 179) que adviene simultáneamente al poder disciplinario y que está focalizado más que en el cuerpo en la mente, en las facultades superiores, en los procesos mentales, en el orden interior. Claro que sin duda aunque el cuerpo se desplace, continúa siendo foco, blanco de distintos poderes y saberes. (Cfr. Le Breton, 2002).

\section{Educación motriz}

Decroly en su libro El niño anormal (1934) precisará las fases en que puede distinguirse, naturalmente, el tratamiento educativo: educación sensorial, educación motriz, educación afectiva, educación intelectual, educación del lenguaje, preparación y orientación profesional. Este tratamiento es diferente del tratamiento médico, pero a su parecer, "la mayor parte de los ejercicios físicos, y principalmente la gimnasia, corresponde tanto al médico como al pedagogo" (Op. cit., 94).
La educación motriz implica la educación de los sentidos. Decroly dista del pensamiento sensualista de Locke (imperante en la primera mitad del siglo XIX) o de Detust de Tracy y se ubica más en la mirada de la naciente psicología experimental pragmática de inicios del siglo XX (James, Wundt): las sensaciones y los sentidos son externos e internos, la actividad es interna, el sujeto es activo en el acto de conocimiento (Véase Sáenz, 1999; Sáenz, Saldarriaga y Ospina, 1997). Además, se basa en los postulados de la psicogénesis para demostrar que los sentidos se forman espontáneamente en la actividad y no es necesaria una educación programática para que el niño los desarrolle. La educación de los sentidos es, en realidad, sobre todo una educación de la atención, de la observación, del vocabulario, de la conciencia, de los hábitos (Decroly, 1934, 95). Esta educación consistiría en la organización de los medios y los objetos de forma tal que los niños anormales se puedan desarrollar, evolucionar a través de la actividad.

La educación motriz del anormal concibe como inherente la unión entre la actividad de los sentidos y la actividad mental. La diferencia tangencial entre exterior e interior se diluye en la pedagogía decrolyana. Movimiento del cuerpo y actividad mental se retroalimentan, se condicionan, se encuentran ligados sin discusión.

Desde el punto de vista biológico, el movimiento es naturalmente la respuesta a un estimulante [...] El movimiento que responderá mejor a las condiciones mentales será el más fecundo como rendimiento y adaptación, el más apropiado para mejorar las condiciones del movimiento mismo. Así, el niño, en el momento en que desea coger una cosa, llega sin ejercicios especiales sugeridos por nadie a ejecutar poco a poco los movimientos necesarios, con una precisión cada vez mayor y un resultado más perfecto. Es que tiene deseo de manipular, de experimentar, que estimula las repeticiones sin fin y la comprobación de la eficacia del esfuerzo hecho. (Decroly, 1934, 97)

Se deja ver pues la fuerte influencia biomédica y experimental subyacente a su pensamiento pedagógico: la vida, el bios, demarca la educación, la actividad mental, los intereses, las aptitudes naturales. La educación de la motricidad, del cuerpo, es educación para la vida y por la vida, en tanto la vida humana es orgánica, social, mental (y para nuestra formación social sería también moral).

Lo anterior fluye sin dificultad cuando se piensa en los niños normales, pero en los anormales o irregulares adquiere un matiz distinto. Decroly plantea que no sólo importarán los masajes, los tratamientos físicos (aplicados por los médicos o enfermeras), la gimnasia 
pasiva o activa, e incluso la electroterapia; sino que rescatará la importancia de incluir en la educación motriz la satisfacción de las necesidades naturales de los niños anormales: necesidad de moverse, de jugar, de imitar, el amor propio, la emulación, protegerse de la lluvia, explorar los objetos, etc.

La educación motriz comprende muchos aspectos, según la etapa de desarrollo en que el niño se halle, la extensión y el grado de la insuficiencia. Puede hacerse que los movimientos necesarios para masticar [...] para beber en un vaso [...] para separar un objeto, sean imposibles o muy difíciles, estando desde luego más o menos normal la inteligencia, los sentidos y el carácter. Puede hacerse también que se encuentre sólo en presencia de cierta incapacidad [...] En todos estos casos, la regla es la siguiente: descubrir o despertar necesidades, hacer aplicar los movimientos posibles a la satisfacción de estas necesidades y aumentar poco a poco las dificultades a medida que los movimientos se hacen fáciles (Ibíd., 98-99).

En este punto es donde puede redimensionarse la participación o el papel de la gimnasia o la gimnástica eurítmica: la educación física condicionada a la satisfacción de las necesidades del niño anormal. No será un régimen distanciado e ignorante de las experiencias cotidianas y vitales del cuerpo, el alma y la mente anormal, sino que estará atravesado por lo necesario, lo indispensable para vivir. Sin embargo, esas supuestas necesidades no dejan de ser invenciones modernas de los saberes y poderes biomédico, psicológico y pedagógico. Pensar que las necesidades deban orientar la educación física, a partir del supuesto de que son naturales e inherentes al niño anormal, es basarse en una aporía.

De igual forma, la dominación del cuerpo no será tanto según los de afuera, los externos al individuo, sino que se construirá un espejismo de naturalidad sobre lo que se considera necesario y, por tanto, se creerá y creará que el niño elige, que decide de acuerdo con sus necesidades. Además, diluir la dicotomía entre exterior e interior, movimientos y actividad mental, anclando el uno en lo otro ( $y$ viceversa) permitirá hacer más eficaces, sutiles y soterrados los ejercicios del poder. Éste será precisamente uno de los grandes problemas de las pedagogías correctivas, al decir de Julia Varela (1995, 175): [...] cómo conseguir un nuevo control menos visible, menos opresivo y más operatorio. Para lograrlo, no sólo sitúan al niño en el centro del proceso educativo mismo [...] sino que además hacen coincidir un medio educativo 'artificial', minuciosamente organizado y preparado, con unas supuestas 'necesidades naturales' del niño".

\section{Centros de interés}

El Programa de Centros de Interés puede sintetizarse en tres pasos o actividades: observación, asociación y expresión. La idea centro se desarrolla a través de este procedimiento, teniendo en cuenta las necesidades primarias (alimentarse, luchar contra las inclemencias del clima, defenderse de peligros y accidentes diversos y buscar en la acción y el trabajo la renovación constante y la alegría del espíritu; Restrepo Mejía, S. F., 1930, 127) y los medios natural o viviente, inanimado y social. Entre necesidades y medios se eligen naturalmente los centros de interés que desarrollarán tanto las facultades intelectuales como el cuerpo, los sentidos del niño, los comportamientos morales y la disposición para los trabajos, en suma: para la vida moderna.

La intervención de los sentidos y de la experiencia se usaba, sobre todo, en la observación y en la expresión, puesto que la asociación es concebida como actividad netamente mental. Los ejercicios de los sentidos estaban motivados por el interés y la necesidad. Por ejemplo, en un centro de interés que gire alrededor del frío o las intemperies (Decroly y Boon, 1927, 41-47) la forma en que se utilizan los sentidos puede ser variada: salir al campo para observar las plantas y los animales comparados con el niño; agrupar los animales y plantas de acuerdo con la forma en que se protejan contra el frío; observar y sentir las sensaciones de estar en campo abierto probando trajes confeccionados con varias telas y después asociar y comparar con los animales; construir gráficos con dibujos y objetos. Otro ejemplo puede ser el correspondiente al "fuego".

Verdadera monografia del fuego. El niño habla desordenadamente, sin el cliché de la respuesta pedagógica, delante del cartón que contiene la síntesis coloreada del motivo. Cultivo ejemplar de la vista que describe cuidadosamente y buen ejercicio de la imaginación, porque la sala se puebla de la escena lejana hecha presente: forja o mina. En torno del fuego, el elemento bello por excelencia, se agrupan los oficios del fuego: el deshollinador, el herrero, la cocinera. Y como 1 materia sólo es preciosa para el niño cuando arde, o se funda, o se exhala en olor: aquí está también la pequeña cocina donde los niños encienden el fuego, y manejan unas marmitas de casa de muñecas: $y$ aquí los fogones de barro que ellos construyen. La escuela Decroly es utilitaria, por realista (Mistral, 1927, 269).

Los casos concretos sobre centros de interés podrían proliferar. El método por excelencia de la pedagogía decrolyana incorpora un modo de normalización del 
cuerpo anormal mediante la educación de los sentidos, lo cual implica, sin duda, una educación normalizadora de la mente, del alma, del espíritu.

\section{Juegos educativos}

Los juegos educativos funcionaban dentro del método de enseñanza decrolyano como una pieza fundamental para el trabajo del cuerpo. Sin lugar a dudas, todo movimiento y toda actividad proporcionaban cultivo físico y, por tanto, intelectual y moral; aunque en la mayoría de los casos debían complementarse mutuamente. El juego educativo se constituye en una de las formas naturales de moldear, de normalizar la corporalidad anormal. Se presenta como natural y constitutivo al desarrollo del niño. Sus leyes lo determinan como propio al infante.

Cuando la pedagogía activa de corte experimental apropiada en Colombia se contrapone a la pedagogía tradicional o clásica (identificada con el "pestalozzianismo perfeccionado" y la pedagogía oficial confesional; para ampliar, veáse Quiceno, 2004; Sáenz, Saldarriaga y Ospina, 1997) esgrima, entre otros, los siguientes argumentos: el memorismo, la repetición centrada en el saber enciclopédico, en lo intelectual; pero la crítica más contundente estaba en desvelar su actividad pasiva. Mediante un conjunto de operaciones diversas, instala al niño en el centro de la práctica pedagógica y valora enormemente el aprender sobre el enseñar, tanto así que los métodos de enseñanza deberán estar determinados por el interés y el aprendizaje del niño anormal y la infancia en general.

De esa manera, con la naturalización del niño, la actividad, el aprendizaje y el interés como ejes de la práctica pedagógica, se incorpora en el saber pedagógico el juego educativo de Decroly como un modo de naturalizar las prácticas efectuadas en una artificialidad inventada (la escuela de anormales y las escuelas de educación pública); esta operación se realizó sostenida en la legitimidad otorgada por los postulados del positivismo evolucionista, por la biomedicina, la biología, la psicología experimental y la pedagogía activa misma (James, Wundt, Pieron, Buyse, Binet, Demoor, entre otros). La enseñanza se presenta como más atractiva, más activa, más acorde a las etapas del desarrollo del niño, en tanto se fundamenta en el simulacro de tomar en cuenta las aptitudes, los dones naturales y los intereses del anormal y el normal; razonamiento dentro del cual se incrustan perfectamente los juegos educativos.

Los juegos educativos eran variados y cobijaban casi todas las facultades, actividades y movimientos del niño: juegos para el desarrollo de las percepciones motrices y la aptitud motriz, juegos visuales (de colores y de formas), juegos visuales motores, juegos auditivo-motores, juegos de iniciación aritmética, referidos a la noción de tiempo, juegos de iniciación a la lectura, juegos de gramática y comprensión del lenguaje. Evocaremos sólo los directamente implicados en la educación motriz o física.

Los juegos de percepción y aptitud motriz se dividen en: visuales, visuales-motores, motores, y auditivo-motores. Estos juegos [...] más que desarrollar los sentidos, enseñan al niño a registrar sus impresiones, a clasificarlas, combinarlas y asociarlas con otras. Además, provocan su atención voluntaria sobre las cualidades de los objetos; le hacen adquirir conciencia de cuanto cae bajo el dominio de sus sentidos, formar su juicio y actuar según las conclusiones de éste». (Decroly y Monchamp, $1928,19)$. No tanto directamente el cuerpo como sí la actividad mental, pero mediante el ejercicio sobre el cuerpo. Todo el batallón de técnicas se desplegaba con carácter de juegos educativos: "Las pompas de jabón", "la pelota", "la niña de paseo", "los buenos amigos", "los objetos coloreados", "las escenas coloreadas", "juegos de paciencia", "juego de escenas incompletas", "los zuecos", "los jarros", "clasificaciones combinadas", "los objetos que faltan", "los vasos", "el gimnasta”, etc.

En últimas, un eufemismo o espejismo de lo natural impera en estos métodos decrolyanos en los que se muestra la educación sensorial de las corporalidades anormales. El cuerpo anormal será educado mediante la educación motriz, los centros de interés y los juegos educativos. El objetivo último está formulado desde el comienzo: educación por la vida y para la vida. Pero, ¿la sociedad moderna disponía las condiciones para que esa educación de la vida natural y social fuera equitativa y democrática? Más allá: ¿por qué designaba como imperativo que se educaran hasta los "anormales"? El poder se hace ver ante nuestros ojos con su rostro amorfo y risueño diciendo: "Hasta los cuerpos y almas más anormales deben producir, nadie se escapa a mi ejercicio, y si piensan que pueden hacerlo, estoy un paso delante de ustedes, posibilitando que piensen que lo artificial es natural, que los intereses son suyos, que el niño es el centro de la pedagogía, que aprender es lo más importante". La educación o pedagogía de anormales es una forma de normalización, disciplinamiento y agenciamiento de los sujetos y las poblaciones anormales. Ni siquiera el cuerpo de los anormales puede escaparse, huirle al poder disciplinario, al biopoder o al psicopoder. No obstante, pueden pensarse otras prácticas desde un lugar distinto. 


\section{Bibliografía}

ÁLVAREZ URÍA, Fernando. (1996). La configuración del campo de la infancia anormal. De la genealogía foucaultiana y de su aplicación a las instituciones de educación especial. En FRANKLIN, Barry. Interpretación de la discapacidad. Teoría e historia de la educación especial. Barcelona-España. Ediciones Pomares-Corredor. Pp. 90-122

BOON, Gérard. (1927). Hacia la escuela renovada. Una primera etapa. España. Ediciones de la Lectura.

CEREZO MANRIQUE, Miguel Ángel. (2003). Los inicios de la psicopedagogía en España. En OSSENBACH SAUTER, Gabriela (Coordinación) Psicología y pedagogía en la primera mitad del siglo XX. Madrid, España. UNED Ediciones.

DECROLY, Ovidio. (1934). El niño anormal: estudios pedagógicos y psicológicos. España. Francisco Beltrán.

DUBREUCQ, Francine. (1993). Jean Ovide Decroly. En Pensadores de la educación. Perspectivas. Revista Trimestral de Educación. V.23, No. 1-2 (85-86). Chile. Unesco. pp. 261-287.

HERRÁIZ GASCUEÑA, Nariano G. (1996). La conceptualización de la infancia deficiente en los inicios de la educación especial europea. En Revista Española de Pedagogía, Año LIV, No. 203. Madrid, España. pp. 167-179.

LE BRETON, David. (2002). Antropología del cuerpo y modernidad. Buenos Aires, Argentina. Ediciones Nueva Visión.

LONDOÑO RAMOS, Carlos Arturo. (2001). La escuela para la vida y por la vida: el impacto de Ovidio Decroly en la pedagogía y la universidad Colombiana". En Revista Historia de la Educación Colombiana. Nos. 3 y 4 . Pereira, Colombia. pp. 135-146.

MISTRAL, Gabriela. (1927). El método Decroly. En: Letras y Encajes, No. 16. Medellín, Colombia. pp. 268-269.

MONCHAMP, Mlle. (1928). La iniciación a la actividad intelectual y motriz por los juegos educativos. Contribución a la pedagogía de los niños y de los irregulares. Madrid, España. Francisco Beltrán, Librería Española y Extranjera.

MUEL, Francine. (1991). La escuela obligatoria y la invención de la infancia anormal. En: CASTEL, Robert; DONZELOT, Jacques; FOUCAULT, Michel; DE GAUDEMAR, Jean-Paul ; GRIGNON, Claude; y MUEL, Francine. Espacios de poder. España. Ediciones de La Piqueta.

QUICENO CASTRILLÓN, Humberto. (2004). Pedagogía católica y escuela activa en Colombia: 1900-1935. Segunda Edición. Bogota, Colombia. Editorial Magisterio.

RESTREPO MEJíA, Martín. (Sin fecha. Aprox. 1930). Pedagogía de párvulos. Exposición de la enseñanza activa. Bogotá, Colombia. Editorial de Cromos.

VARELA, Julia. (1995). Categorías espacio temporales y socialización escolar. Del individualismo al narcisismo. En: LARROSA, Jorge. (Editor). Escuela, poder y subjetivación. Madrid, España. Ediciones de La Piqueta. pp. 155-189

VARELA, Julia. (1991). Arqueología de la Escuela. Madrid, España. Las Ediciones de La Piqueta.

\section{Notas}

1 Por tecnologias médico (psico) pedagógicas se entiende aquel conjunto de técnicas y prácticas dirigidas hacia el gobierno $o$ agenciamiento de la "infancia anormal", la cual pasó a ser objeto-blanco de una interdiscursividad especifica, y, por tanto, se sumergió en un agrupamiento de prácticas encaminadas hacia la vigilancia y el escrutinio del alma y el cuerpo -por una parte- y hacia el control de si mismo con fines progresistas, productivos, morales y de (re) socialización -por otra-. El tipo de gobierno desplegado con este "conjunto de técnicas" se encuentra incrustado en el funcionamiento de dos tecnologias: las de poder y las del sí mismo. Las primeras "determinan la conducta de los individuos, los someten a cierto

tipos de fines o de dominación, y consisten en una objetivación del sujeto" (Foucault, 1991, 48), y las segundas "permiten a los individuos efectuar, por cuenta propia o con la ayuda de otros, cierto número de operaciones sobre su cuerpo y su alma, pensamientos, conducta, o cualquier forma de ser, obteniendo así una transformación de sí mismos con el fin de alcanzar cierto estado de felicidad, pureza, sabiduría o inmortalidad" (Ibid.)

2 Veáse SÁENZ OBREGÓN, Javier (1999) Ovidio Decroly. Educabilidad, naturaleza y método. En Maestros Pedagogos II. Un diálogo con el presente. Corporación Región. Medellin, Colombia. pp. 53-70. 